\title{
A Lower Bound of the First Dirichlet Eigenvalue of a Compact Manifold with Positive Ricci Curvature*
}

\author{
Jun LING
}

\begin{abstract}
We give a new estimate on the lower bound for the first Dirichlet eigenvalue for a compact manifold with positive Ricci curvature in terms of the in-diameter and the lower bound of the Ricci curvature. The result improves the previous estimates.
\end{abstract}

\section{Introduction}

If $(M, g)$ is an n-dimensional compact Riemannian manifold whose Ricci curvature has a positive lower bound $(n-1) K$ for some constant $K>0$ and whose non-empty boundary $\partial M$ has nonnegative mean curvature with respect to the outward normal, Reilly [10] gave the following lower bound of the first Dirichlet eigenvalue $\lambda$ of the Laplacian on $M$

$$
\lambda \geq n K \text {. }
$$

This estimate gives no information when the above constant $K$ vanishes. In such case, Li-Yau [5] and Zhong-Yang [14] provided another lower bound for the first non-zero eigenvalue of a closed manifold

$$
\lambda \geq \frac{\pi^{2}}{d^{2}}
$$

It is an interesting problem to find a unified lower bound of the first Dirichlet eigenvalue $\lambda$ in terms of the lower bound $(n-1) K$ of the Ricci curvature and the diameter $d$, in-diameter $\tilde{d}$ and other geometric quantities, which do not vanish as $K$ vanishes, of the manifold with positive Ricci curvature. D. Yang [12] proved that

$$
\lambda \geq \frac{1}{4}(n-1) K+\frac{\pi^{2}}{(\tilde{d})^{2}},
$$

*2000 Mathematics Subject Classification Primary 58J50, 35P15; Secondary 53C21 
where $\tilde{d}$ is the diameter of the largest interior ball in $M$.

In this paper we give a new estimate on the lower bound of the first Dirichlet eigenvalue $\lambda$. We have the following result.

Theorem 1. If $(M, g)$ is an n-dimensional compact Riemannian manifold with boundary. Suppose that Ricci curvature $\operatorname{Ric}(M)$ of $M$ is bounded below by $(n-1) K$ for some constant $K>0$

$$
\operatorname{Ric}(M) \geq(n-1) K
$$

and that the mean curvature of the boundary $\partial M$ with respect to the outward normal is nonnegative, then the first Dirichlet eigenvalue $\lambda$ of the Laplacian $\Delta$ of $M$ has the following lower bound

$$
\lambda \geq \frac{1}{2}(n-1) K+\frac{\pi^{2}}{(\tilde{d})^{2}},
$$

where $\tilde{d}$ is the diameter of the largest interior ball in $M$, that is, $\tilde{d}=$ $2 \sup _{x \in M}\{\operatorname{dist}(x, \partial M)\}$.

Our result improves Yang' bound (2) by doubling the coefficient before $(n-1) K$. In the proof, we use a function $\xi$ that the author constructed in 8 ] for the construction of the suitable test function instead of using the ZhongYang's canonical function. That provides a new way to sharpen the bound. In the next section, we derive some preliminary estimates and conditions for test functions first and we construct the needed test function and prove the main result in the last section.

\section{Preliminary Estimates}

The first basic estimate is of Lichnerowicz-type. Recall that the classic Lichnerowicz Theorem [6] states that if $M$ is an $n$-dimensional closed manifold whose Ricci curvature satisfies (3) then the first non-zero eigenvalue has a lower bound (11). Reilly [10] proved that this Lichnerowicz-type estimate remains true for the first Dirichlet eigenvalue $\lambda$ as well if the manifold has the same lower bound for the Ricci curvature and has non-empty boundary whose mean curvature with respect to the outward normal is nonnegative. For the completeness and consistency, we use gradient estimate in [2]-[5] and [11] to derive the Lichnerowicz-type estimate.

Lemma 1. Under the conditions in Theorem 1, the estimate (1) holds. 
Proof. Let $v$ be a normalized eigenfunction of the first Dirichlet eigenvalue such that

$$
\sup _{M} v=1, \quad \inf _{M} v=0 .
$$

The function $v$ satisfies the following

$$
\Delta v=-\lambda v \text { in } M
$$

and

$$
v=0 \quad \text { on } \partial M \text {. }
$$

Take an orthonormal frame $\left\{e_{1}, \ldots, e_{n}\right\}$ of $M$ about $x_{0} \in M$. At $x_{0}$ we have

$$
\nabla_{e_{j}}\left(|\nabla v|^{2}\right)\left(x_{0}\right)=\sum_{i=1}^{n} 2 v_{i} v_{i j}
$$

and

$$
\begin{aligned}
\Delta\left(|\nabla v|^{2}\right)\left(x_{0}\right) & =2 \sum_{i, j=1}^{n} v_{i j} v_{i j}+2 \sum_{i, j=1}^{n} v_{i} v_{i j j} \\
& =2 \sum_{i, j=1}^{n} v_{i j} v_{i j}+2 \sum_{i, j=1}^{n} v_{i} v_{j j i}+2 \sum_{i, j=1}^{n} \mathrm{R}_{i j} v_{i} v_{j} \\
& =2 \sum_{i, j=1}^{n} v_{i j} v_{i j}+2 \nabla v \nabla(\Delta v)+2 \operatorname{Ric}(\nabla v, \nabla v) \\
& \geq 2 \sum_{i=1}^{n} v_{i i}^{2}+2 \nabla v \nabla(\Delta v)+2(n-1) K|\nabla v|^{2} \\
& \geq \frac{2}{n}(\Delta v)^{2}-2 \lambda|\nabla v|^{2}+2(n-1) K|\nabla v|^{2} .
\end{aligned}
$$

Thus at all point $x \in M$,

$$
\frac{1}{2} \Delta\left(|\nabla v|^{2}\right) \geq \frac{1}{n} \lambda^{2} v^{2}+[(n-1) K-\lambda]|\nabla v|^{2} .
$$

On the other hand, after multiplying (6) by $v$ and integrating both sides over $M$ and using (7), we have

$$
\int_{M} \lambda v^{2} d x=-\int_{M} v \Delta v d x
$$




$$
=-\int_{\partial M} v \frac{\partial}{\partial \nu} v d s+\int_{M}|\nabla v|^{2} d x=\int_{M}|\nabla v|^{2} d x,
$$

where and below $\nu$ is the outward normal of $\partial M$. That the integral on the boundary vanishes is due to (7). Integrating (8) over $M$ and using the above equality, we get

$$
\frac{1}{2} \int_{\partial M} \frac{\partial}{\partial \nu}\left(|\nabla v|^{2}\right) d x \geq \int_{M}(n K-\lambda) \frac{n-1}{n} \lambda v^{2} d x .
$$

We need show that $\frac{\partial}{\partial \nu}\left(|\nabla v|^{2}\right) \leq 0$ on $\partial M$. Take any $x_{0} \in \partial M$. If $\nabla v\left(x_{0}\right)=0$, then it is done. Assume now that $\nabla v\left(x_{0}\right) \neq 0$. Choose a local orthonormal frame $\left\{e_{1}, e_{2}, \cdots, e_{n}\right\}$ of $M$ about $x_{0}$ so that $e_{n}$ is the unit outward normal vector field near $x_{0} \in \partial M$ and $\left.\left\{e_{1}, e_{2}, \cdots, e_{n-1}\right\}\right|_{\partial M}$ is a local frame of $\partial M$ about $x_{0}$. The existence of such local frame can be justified as the following. Let $e_{n}$ be the local unit outward normal vector field of $\partial M$ about $x_{0} \in \partial M$ and $\left\{e_{1}, \cdots, e_{n-1}\right\}$ the local orthonormal frame of $\partial M$ about $x_{0}$. By parallel translation along the geodesic $\gamma(t)=\exp _{x_{0}} t e_{n}$, we may extend $e_{1}, \cdots, e_{n-1}$ to local vector fields of $M$. Then the extended frame $\left\{e_{1}, e_{2}, \cdots, e_{n}\right\}$ is what we need. Note that $\nabla_{e_{n}} e_{i}=0$ for $i \leq n-1$. Since $\left.v\right|_{\partial M}=0$, we have $v_{i}\left(x_{0}\right)=0$ for $i \leq n-1$. Using (5)-(7) in the following arguments, then we have that at $x_{0}$,

$$
\begin{aligned}
& \frac{\partial}{\partial \nu}\left(|\nabla v|^{2}\right)\left(x_{0}\right)=\sum_{i=1}^{n} 2 v_{i} v_{i n}=2 v_{n} v_{n n} \\
& =2 v_{n}\left(\Delta^{M} v-\sum_{i=1}^{n-1} v_{i i}\right)=2 v_{n}\left(-\lambda v-\sum_{i=1}^{n-1} v_{i i}\right) \\
& =-2 v_{n} \sum_{i=1}^{n-1} v_{i i}=-2 v_{n} \sum_{i=1}^{n-1}\left(e_{i} e_{i} v-\nabla_{e_{i}}^{M} e_{i} v\right) \\
& =2 v_{n} \sum_{i=1}^{n-1} \nabla_{e_{i}}^{M} e_{i} v=2 v_{n} \sum_{i=1}^{n-1} \sum_{j=1}^{n} g\left(\nabla_{e_{i}}^{M} e_{i}, e_{j}\right) v_{j} \\
& =2 v_{n}^{2} \sum_{i=1}^{n-1} g\left(\nabla_{e_{i}}^{M} e_{i}, e_{n}\right)=-2 v_{n}^{2} \sum_{i=1}^{n-1} g\left(\nabla_{e_{i}}^{M} e_{n}, e_{i}\right) \\
& =-2 v_{n}^{2} \sum_{i=1}^{n-1} h_{i i}=-2 v_{n}^{2}\left(x_{0}\right) m\left(x_{0}\right)
\end{aligned}
$$

$\leq 0$ by the non-negativity of $m$, 
where $g($,$) is the Riemann metric of M,\left(h_{i j}\right)$ is the second fundamental form of $\partial M$ with respect to the outward normal $\nu$ and $m$ is the mean curvature of $\partial M$ with respect to $\nu$. Therefore (1) holds.

Lemma 2. Let $v$ be, as the above, the normalized eigenfunction for the first Dirichlet eigenvalue $\lambda$. Then $v$ satisfies the following

$$
\frac{|\nabla v|^{2}}{b^{2}-v^{2}} \leq \lambda
$$

where $b>1$ is an arbitrary constant.

Proof. Consider the function

$$
P(x)=|\nabla v|^{2}+A v^{2},
$$

where $A=\lambda(1+\epsilon)$ for small $\epsilon>0$. Function $P$ must achieve its maximum at some point $x_{0} \in M$. We claim that

$$
\nabla P\left(x_{0}\right)=0
$$

If $x_{0} \in M \backslash \partial M$, (13) is obviously true. Suppose that $x_{0} \in \partial M$. Take the same local orthonormal frame $\left\{e_{1}, e_{2}, \cdots, e_{n}\right\}$ of $M$ about $x_{0}$ as in the proof of Lemma 1 where $e_{n}$ is the unit outward normal vector field near $x_{0} \in \partial M$, $\left.\left\{e_{1}, e_{2}, \cdots, e_{n-1}\right\}\right|_{\partial M}$ is a local frame of $\partial M$ about $x_{0}$ and $\nabla_{e_{n}} e_{i}=0$ for $i \leq n-1$. Since $\left.v\right|_{\partial M}=0$, we have $v_{i}\left(x_{0}\right)=0$ for $i \leq n-1$. $P\left(x_{0}\right)$ is the maximum implies that

$$
P_{i}\left(x_{0}\right)=0 \quad \text { for } i \leq n-1
$$

and

$$
P_{n}\left(x_{0}\right) \geq 0 .
$$

Using argument in proving (10) and the non-negativity of the mean curvature $m$ of $\partial M$ with respect to the outward normal, we get

$$
\nabla_{e_{n}}\left(|\nabla v|^{2}\right)\left(x_{0}\right) \leq 0 .
$$

Noticing that $\left.v\right|_{\partial M}=0$, we have

$$
\left.P_{n}\left(x_{0}\right)=\nabla_{e_{n}}\left(|\nabla v|^{2}\right)\left(x_{0}\right)+2 A v\left(x_{0}\right) v_{n}\left(x_{0}\right)\right) \leq 0 .
$$

Now (14), (15) and (16) imply that $P_{n}\left(x_{0}\right)=0$ and $\nabla P\left(x_{0}\right)=0$. 
Thus (13) holds, no matter $x_{0} \notin \partial M$ or $x_{0} \in \partial M$. By (13) and the Maximum Principle, we have

$$
\nabla P\left(x_{0}\right)=0 \quad \text { and } \quad \Delta P\left(x_{0}\right) \leq 0 .
$$

We are going to show further that $\nabla v\left(x_{0}\right)=0$. If on the contrary, $\nabla v\left(x_{0}\right) \neq 0$, then we rotate the local orthonormal frame about $x_{0}$ such that

$$
\left|v_{1}\left(x_{0}\right)\right|=\left|\nabla v\left(x_{0}\right)\right| \neq 0 \quad \text { and } \quad v_{i}\left(x_{0}\right)=0, \quad i \geq 2 .
$$

From (17) we have at $x_{0}$,

$$
\begin{gathered}
0=\frac{1}{2} \nabla_{i} P=\sum_{j=1}^{n} v_{j} v_{j i}+A v v_{i}, \\
v_{11}=-A v \quad \text { and } \quad v_{1 i}=0 \quad i \geq 2,
\end{gathered}
$$

and

$$
\begin{aligned}
0 & \geq \frac{1}{2} \Delta P\left(x_{0}\right)=\sum_{i, j=1}^{n}\left(v_{j i} v_{j i}+v_{j} v_{j i i}+A v_{i} v_{i}+A v v_{i i}\right) \\
& =\sum_{i, j=1}^{n}\left(v_{j i}^{2}+v_{j}\left(v_{i i}\right)_{j}+\mathrm{R}_{j i} v_{j} v_{i}+A v_{i i}^{2}+A v v_{i i}\right) \\
& =\sum_{i, j=1}^{n} v_{j i}^{2}+\nabla v \nabla(\Delta v)+\operatorname{Ric}(\nabla v, \nabla v)+A|\nabla v|^{2}+A v \Delta v \\
& \geq v_{11}^{2}+\nabla v \nabla(\Delta v)+(n-1) K|\nabla v|^{2}+A|\nabla v|^{2}+A v \Delta v \\
& =(-A v)^{2}-\lambda|\nabla v|^{2}+(n-1) K|\nabla v|^{2}+A|\nabla v|^{2}-\lambda A v^{2} \\
& =(A-\lambda+(n-1) K)|\nabla v|^{2}+A v^{2}(A-\lambda),
\end{aligned}
$$

where we have used (18) and (3). Therefore at $x_{0}$,

$$
0 \geq(A-\lambda)|\nabla v|^{2}+A(A-\lambda) v^{2},
$$

that is,

$$
\left|\nabla v\left(x_{0}\right)\right|^{2}+\lambda(1+\epsilon) v\left(x_{0}\right)^{2} \leq 0 .
$$

Thus $\nabla v\left(x_{0}\right)=0$. This contradicts $\nabla v\left(x_{0}\right) \neq 0$. 
Therefore in any case, if $P$ achieves its maximum at a point $x_{0}$, then $\nabla v\left(x_{0}\right)=0$. Thus at $x_{0}$

$$
P\left(x_{0}\right)=\left|\nabla v\left(x_{0}\right)\right|^{2}+A v\left(x_{0}\right)^{2}=A v\left(x_{0}\right)^{2} \leq A .
$$

and at all $x \in M$

$$
|\nabla v(x)|^{2}+A v(x)^{2}=P(x) \leq P\left(x_{0}\right) \leq A .
$$

Letting $\epsilon \rightarrow 0$ in the above inequality, the estimate (11) follows.

We want to improve the upper bound in (11) further and proceed in the following way.

Define a function $F$ by

$$
Z(t)=\max _{x \in M, t=\sin ^{-1}(v(x) / b)} \frac{|\nabla v|^{2}}{b^{2}-v^{2}} / \lambda .
$$

The estimate in (11) becomes

$$
Z(t) \leq 1 \quad \text { on }\left[0, \sin ^{-1}(1 / b)\right]
$$

For convenience, in this paper we let

$$
\alpha=\frac{1}{2}(n-1) K \quad \text { and } \quad \delta=\alpha / \lambda .
$$

By (11) we have

$$
\delta \leq \frac{n-1}{2 n} .
$$

We have the following conditions for the test function $Z$.

Theorem 2. If the function $z:\left[0, \sin ^{-1}(1 / b)\right] \mapsto \mathbf{R}^{1}$ satisfies the following

1. $z(t) \geq Z(t) \quad t \in\left[0, \sin ^{-1}(1 / b)\right]$,

2. there exists some $x_{0} \in M$ such that at point $t_{0}=\sin ^{-1}\left(v\left(x_{0}\right) / b\right)$ $z\left(t_{0}\right)=Z\left(t_{0}\right)$

3. $z\left(t_{0}\right)>0$,

4. $z$ extends to a smooth even function, and

5. $z^{\prime}\left(t_{0}\right) \sin t_{0} \geq 0$, 
then we have the following

$$
0 \leq \frac{1}{2} z^{\prime \prime}\left(t_{0}\right) \cos ^{2} t_{0}-z^{\prime}\left(t_{0}\right) \cos t_{0} \sin t_{0}-z\left(t_{0}\right)+1-2 \delta \cos ^{2} t_{0} .
$$

Proof. Define

$$
J(x)=\left\{\frac{|\nabla v|^{2}}{b^{2}-v^{2}}-\lambda z\right\} \cos ^{2} t,
$$

where $t=\sin ^{-1}(v(x) / b)$. Then

$$
J(x) \leq 0 \quad \text { for } x \in M \quad \text { and } \quad J\left(x_{0}\right)=0 .
$$

So $J\left(x_{0}\right)$ is the maximum of $J$ on $M$. If $\nabla v\left(x_{0}\right)=0$, then

$$
0=J\left(x_{0}\right)=-\lambda z \cos ^{2} t .
$$

This contradicts the Condition 3 in the theorem. Therefore

$$
\nabla v\left(x_{0}\right) \neq 0
$$

We claim that

$$
\nabla J\left(x_{0}\right)=0
$$

If $x_{0} \in M \backslash \partial M$, (24) is obviously true. Suppose that $x_{0} \in \partial M$. Take the same local orthonormal frame $\left\{e_{1}, e_{2}, \cdots, e_{n}\right\}$ of $M$ about $x_{0}$ as in the proof of Lemma1, where $e_{n}$ is the unit outward normal vector field near $x_{0} \in \partial M$, $\left.\left\{e_{1}, e_{2}, \cdots, e_{n-1}\right\}\right|_{\partial M}$ is a local frame of $\partial M$ about $x_{0}$ and $\nabla_{e_{n}} e_{i}=0$ for $i \leq n-1$. Since $\left.v\right|_{\partial M}=0$, we have $v_{i}\left(x_{0}\right)=0$ for $i \leq n-1$. $J\left(x_{0}\right)$ is the maximum implies that

$$
J_{i}\left(x_{0}\right)=0 \quad \text { for } i \leq n-1
$$

and

$$
J_{n}\left(x_{0}\right) \geq 0 .
$$

Using argument in proving (10) and the non-negativity of the mean curvature $m$ of $\partial M$ with respect to the outward normal, we get

$$
\left.\left(|\nabla v|^{2}\right)_{n}\right|_{x_{0}} \leq 0
$$


The Dirichlet condition $v\left(x_{0}\right)=0$ implies that $t\left(x_{0}\right)=0$ and $z^{\prime}\left(t\left(x_{0}\right)\right)=$ $z^{\prime}(0)=0$, since by the Condition 4 in the theorem $z$ extends to a smooth even function. Therefore

$J_{n}\left(x_{0}\right)=\frac{1}{b^{2}}\left(|\nabla v|^{2}\right)_{n}-\left.\lambda \cos t\left[z^{\prime} \cos t-2 z \sin t\right] t_{n}\right|_{x_{0}}=\left.\frac{1}{b^{2}}\left(|\nabla v|^{2}\right)_{n}\right|_{x_{0}} \leq 0$.

Now (25), (26) and (27) imply (24).

Thus (24) holds, no matter $x_{0} \notin \partial M$ or $x_{0} \in \partial M$. By (24) and the Maximum Principle, we have

$$
\nabla J\left(x_{0}\right)=0 \quad \text { and } \quad \Delta J\left(x_{0}\right) \leq 0 .
$$

$J(x)$ can be rewritten as

$$
J(x)=\frac{1}{b^{2}}|\nabla v|^{2}-\lambda z \cos ^{2} t .
$$

Thus (28) is equivalent to

$$
\left.\frac{2}{b^{2}} \sum_{i} v_{i} v_{i j}\right|_{x_{0}}=\left.\lambda \cos t\left[z^{\prime} \cos t-2 z \sin t\right] t_{j}\right|_{x_{0}}
$$

and

$$
\begin{aligned}
0 \geq & \frac{2}{b^{2}} \sum_{i, j} v_{i j}^{2}+\frac{2}{b^{2}} \sum_{i, j} v_{i} v_{i j j}-\lambda\left(z^{\prime \prime}|\nabla t|^{2}+z^{\prime} \Delta t\right) \cos ^{2} t \\
& +4 \lambda z^{\prime} \cos t \sin t|\nabla t|^{2}-\left.\lambda z \Delta \cos ^{2} t\right|_{x_{0}}
\end{aligned}
$$

Rotate the frame so that

$$
\left|v_{1}\left(x_{0}\right)\right|=\left|\nabla v\left(x_{0}\right)\right| \neq 0 \quad \text { and } \quad v_{i}\left(x_{0}\right)=0, \quad i \geq 2 .
$$

Then (29) implies

$$
\left.v_{11}\right|_{x_{0}}=\left.\frac{\lambda b}{2}\left(z^{\prime} \cos t-2 z \sin t\right)\right|_{x_{0}} \text { and }\left.\quad v_{1 i}\right|_{x_{0}}=0 \text { for } i \geq 2 .
$$


Now we have

$$
\begin{aligned}
\left.|\nabla v|^{2}\right|_{x_{0}} & =\left.\lambda b^{2} z \cos ^{2} t\right|_{x_{0}}, \\
\left.|\nabla t|^{2}\right|_{x_{0}} & =\frac{|\nabla v|^{2}}{b^{2}-v^{2}}=\left.\lambda z\right|_{x_{0}}, \\
\left.\frac{\Delta v}{b}\right|_{x_{0}} & =\Delta \sin t=\cos t \Delta t-\left.\sin t|\nabla t|^{2}\right|_{x_{0}}, \\
\left.\Delta t\right|_{x_{0}} & =\frac{1}{\cos t}\left(\sin t|\nabla t|^{2}+\frac{\Delta v}{b}\right) \\
\left.\Delta \cos ^{2} t\right|_{x_{0}} & =\left.\frac{1}{\cos t}\left[\lambda z \sin t-\frac{\lambda}{b} v\right]\right|_{x_{0}}, \quad \text { and } \\
& =-2 \lambda z \cos ^{2} t+\left.\frac{2}{b^{2}} \lambda v^{2}\right|_{x_{0}} .
\end{aligned}
$$

Therefore,

$$
\begin{gathered}
\left.\frac{2}{b^{2}} \sum_{i, j} v_{i j}^{2}\right|_{x_{0}} \geq \frac{2}{b^{2}} v_{11}^{2} \\
=\frac{\lambda^{2}}{2}\left(z^{\prime}\right)^{2} \cos ^{2} t-2 \lambda^{2} z z^{\prime} \cos t \sin t+\left.2 \lambda^{2} z^{2} \sin ^{2} t\right|_{x_{0}} \\
\left.\frac{2}{b^{2}} \sum_{i, j} v_{i} v_{i j j}\right|_{x_{0}}=\frac{2}{b^{2}}(\nabla v \nabla(\Delta v)+\operatorname{Ric}(\nabla v, \nabla v)) \\
\geq \frac{2}{b^{2}}\left(\nabla v \nabla(\Delta v)+(n-1) K|\nabla v|^{2}\right) \\
=-2 \lambda^{2} z \cos ^{2} t+\left.4 \alpha \lambda z \cos ^{2} t\right|_{x_{0}} \\
-\left.\lambda\left(z^{\prime \prime}|\nabla t|^{2}+z^{\prime} \Delta t\right) \cos ^{2} t\right|_{x_{0}} \\
=-\lambda^{2} z z^{\prime \prime} \cos ^{2} t-\lambda^{2} z z^{\prime} \cos t \sin t \\
+\left.\frac{1}{b} \lambda^{2} z^{\prime} v \cos ^{2}\right|_{x_{0}},
\end{gathered}
$$


and

$$
\begin{aligned}
& 4 \lambda z^{\prime} \cos t \sin t|\nabla t|^{2}-\left.\lambda z \Delta \cos ^{2} t\right|_{x_{0}} \\
& =4 \lambda^{2} z z^{\prime} \cos t \sin t+2 \lambda^{2} z^{2} \cos ^{2} t-\left.\frac{2}{b} \lambda^{2} z v \sin t\right|_{x_{0}} .
\end{aligned}
$$

Putting these results into (30) we get

$$
\begin{aligned}
0 \geq & -\lambda^{2} z z^{\prime \prime} \cos ^{2} t+\frac{\lambda^{2}}{2}\left(z^{\prime}\right)^{2} \cos ^{2} t+\lambda^{2} z^{\prime} \cos t(z \sin t+\sin t) \\
& +2 \lambda^{2} z^{2}-2 \lambda^{2} z+\left.4 \alpha \lambda z \cos ^{2} t\right|_{x_{0}},
\end{aligned}
$$

where we used (31). Now

$$
z\left(t_{0}\right)>0
$$

by the Condition 3 in the theorem. Dividing two sides of (32) by $\left.2 \lambda^{2} z\right|_{x_{0}}$, we have

$$
\begin{aligned}
0 \geq & -\frac{1}{2} z^{\prime \prime}\left(t_{0}\right) \cos ^{2} t_{0}+\frac{1}{2} z^{\prime}\left(t_{0}\right) \cos t_{0}\left(\sin t_{0}+\frac{\sin t_{0}}{z\left(t_{0}\right)}\right)+z\left(t_{0}\right) \\
& -1+2 \delta \cos ^{2} t_{0}+\frac{1}{4 z\left(t_{0}\right)}\left(z^{\prime}\left(t_{0}\right)\right)^{2} \cos ^{2} t_{0} .
\end{aligned}
$$

Therefore,

$$
\begin{aligned}
0 \geq & -\frac{1}{2} z^{\prime \prime}\left(t_{0}\right) \cos ^{2} t_{0}+z^{\prime}\left(t_{0}\right) \cos t_{0} \sin t_{0}+z\left(t_{0}\right)-1+2 \delta \cos ^{2} t_{0} \\
& +\frac{1}{4 z\left(t_{0}\right)}\left(z^{\prime}\left(t_{0}\right)\right)^{2} \cos ^{2} t_{0}+\frac{1}{2} z^{\prime}\left(t_{0}\right) \sin t_{0} \cos t_{0}\left[\frac{1}{z\left(t_{0}\right)}-1\right] .
\end{aligned}
$$

Conditions 1, 2 and 5 in the theorem imply that $0<z\left(t_{0}\right)=Z\left(t_{0}\right) \leq 1$ and $z^{\prime}\left(t_{0}\right) \sin t_{0} \geq 0$. Therefore the last two terms in (34) are nonnegative and (23) follows.

\section{Proof of Main Result}

Proof of Theorem 1, Let

$$
z(t)=1+\delta \xi(t)
$$


where $\xi$ is the functions defined by (45) in Lemma 3. We claim that

$$
Z(t) \leq z(t) \quad \text { for } t \in\left[0, \sin ^{-1}(1 / b)\right] .
$$

Lemma 3 implies that for $t \in\left[0, \sin ^{-1}(1 / b)\right]$, we have the following

$$
\begin{aligned}
& \frac{1}{2} z^{\prime \prime} \cos ^{2} t-z^{\prime} \cos t \sin t-z=-1+2 \delta \cos ^{2} t \\
& z^{\prime}(t) \sin t \geq 0, \\
& z \text { is a smooth even function, } \\
& 0<1-\left(\frac{\pi^{2}}{4}-1\right) \frac{n-1}{2 n} \leq 1-\left(\frac{\pi^{2}}{4}-1\right) \delta=z(0) \leq z(t), \quad \text { and } \\
& z(t) \leq z\left(\frac{\pi}{2}\right)=1 .
\end{aligned}
$$

Let $P \in \mathbf{R}^{1}$ and $t_{0} \in\left[0, \sin ^{-1}(1 / b)\right]$ such that

$$
P=\max _{t \in\left[0, \sin ^{-1}(1 / b)\right]}(Z(t)-z(t))=Z\left(t_{0}\right)-z\left(t_{0}\right) .
$$

Thus

(42) $Z(t) \leq z(t)+P \quad$ for $t \in\left[0, \sin ^{-1}(1 / b)\right] \quad$ and $\quad Z\left(t_{0}\right)=z\left(t_{0}\right)+P$.

Suppose that $P>0$. Then $z+P$ satisfies the conditions in Theorem 2 and therefore satisfies (23). So we have

$$
\begin{aligned}
& z\left(t_{0}\right)+P=Z\left(t_{0}\right) \\
& \leq \frac{1}{2}(z+P)^{\prime \prime}\left(t_{0}\right) \cos ^{2} t_{0}-(z+P)^{\prime}\left(t_{0}\right) \cos t_{0} \sin t_{0}+1-2 \delta \cos ^{2} t_{0} \\
& =\frac{1}{2} z^{\prime \prime}\left(t_{0}\right) \cos ^{2} t_{0}-z^{\prime}\left(t_{0}\right) \cos t_{0} \sin t_{0}+1-2 \delta \cos ^{2} t_{0} \\
& =z\left(t_{0}\right) .
\end{aligned}
$$

This contradicts the assumption $P>0$. Thus $P \leq 0$ and (36) must hold. That means

$$
\sqrt{\lambda} \geq \frac{|\nabla t|}{\sqrt{z(t)}}
$$

Take $q_{1}$ on $M$ such that $v\left(q_{1}\right)=1=\sup _{M} v$ and and $q_{2} \in \partial M$ such that distance $d\left(q_{1}, q_{2}\right)=$ distance $d\left(q_{1}, \partial M\right)$. Let $L$ be the minimum geodesic 
segment between $q_{1}$ and $q_{2}$. We integrate both sides of (43) along $L$ and change variable and let $b \rightarrow 1$. Let $\tilde{d}$ be the diameter of the largest interior ball in $M$. Then

$$
\sqrt{\lambda} \frac{\tilde{d}}{2} \geq \int_{L} \frac{|\nabla t|}{\sqrt{z(t)}} d l=\int_{0}^{\frac{\pi}{2}} \frac{1}{\sqrt{z(t)}} d t \geq \frac{\left(\int_{0}^{\pi / 2} d t\right)^{\frac{3}{2}}}{\left(\int_{0}^{\pi / 2} z(t) d t\right)^{\frac{1}{2}}} \geq\left(\frac{\left(\frac{\pi}{2}\right)^{3}}{\int_{0}^{\pi / 2} z(t) d t}\right)^{\frac{1}{2}} .
$$

Square the two sides. Then

$$
\lambda \geq \frac{\pi^{3}}{2(\tilde{d})^{2} \int_{0}^{\pi / 2} z(t) d t} .
$$

Now

$$
\int_{0}^{\frac{\pi}{2}} z(t) d t=\int_{0}^{\frac{\pi}{2}}[1+\delta \xi(t)] d t=\frac{\pi}{2}(1-\delta)
$$

by (48) in Lemma 3 . That is,

$$
\lambda \geq \frac{\pi^{2}}{(1-\delta)(\tilde{d})^{2}} \quad \text { and } \quad \lambda \geq \frac{1}{2}(n-1) K+\frac{\pi^{2}}{(\tilde{d})^{2}} .
$$

We now present a lemma that is used in the proof of Theorem 1]

Lemma 3. Let

$$
\xi(t)=\frac{\cos ^{2} t+2 t \sin t \cos t+t^{2}-\frac{\pi^{2}}{4}}{\cos ^{2} t} \quad \text { on } \quad\left[-\frac{\pi}{2}, \frac{\pi}{2}\right] .
$$


Then the function $\xi$ satisfies the following

$$
\begin{aligned}
& \frac{1}{2} \xi^{\prime \prime} \cos ^{2} t-\xi^{\prime} \cos t \sin t-\xi=2 \cos ^{2} t \quad \text { in }\left(-\frac{\pi}{2}, \frac{\pi}{2}\right), \\
& \xi^{\prime} \cos t-2 \xi \sin t=4 t \cos t \\
& \int_{0}^{\frac{\pi}{2}} \xi(t) d t=-\frac{\pi}{2} \\
& 1-\frac{\pi^{2}}{4}=\xi(0) \leq \xi(t) \leq \xi\left( \pm \frac{\pi}{2}\right)=0 \quad \text { on }\left[-\frac{\pi}{2}, \frac{\pi}{2}\right], \\
& \xi^{\prime} \text { is increasing on }\left[-\frac{\pi}{2}, \frac{\pi}{2}\right] \text { and } \xi^{\prime}\left( \pm \frac{\pi}{2}\right)= \pm \frac{2 \pi}{3}, \\
& \xi^{\prime}(t)<0 \text { on }\left(-\frac{\pi}{2}, 0\right) \text { and } \xi^{\prime}(t)>0 \text { on }\left(0, \frac{\pi}{2}\right), \\
& \xi^{\prime \prime}\left( \pm \frac{\pi}{2}\right)=2, \xi^{\prime \prime}(0)=2\left(3-\frac{\pi^{2}}{4}\right) \text { and } \xi^{\prime \prime}(t)>0 \text { on }\left[-\frac{\pi}{2}, \frac{\pi}{2}\right], \\
& \left(\frac{\xi^{\prime}(t)}{t}\right)^{\prime}>0 \text { on }(0, \pi / 2) \text { and } 2\left(3-\frac{\pi^{2}}{4}\right) \leq \frac{\xi^{\prime}(t)}{t} \leq \frac{4}{3} \text { on }\left[-\frac{\pi}{2}, \frac{\pi}{2}\right], \\
& \xi^{\prime \prime \prime}\left(\frac{\pi}{2}\right)=\frac{8 \pi}{15}, \xi^{\prime \prime \prime}(t)<0 \text { on }\left(-\frac{\pi}{2}, 0\right) \text { and } \xi^{\prime \prime \prime}(t)>0 \text { on }\left(0, \frac{\pi}{2}\right) .
\end{aligned}
$$

Proof. For convenience, let $q(t)=\xi^{\prime}(t)$, i.e.,

$$
q(t)=\xi^{\prime}(t)=\frac{2\left(2 t \cos t+t^{2} \sin t+\cos ^{2} t \sin t-\frac{\pi^{2}}{4} \sin t\right)}{\cos ^{3} t} .
$$

Equation (46) and the values $\xi\left( \pm \frac{\pi}{2}\right)=0, \xi(0)=1-\frac{\pi^{2}}{4}$ and $\xi^{\prime}\left( \pm \frac{\pi}{2}\right)= \pm \frac{2 \pi}{3}$ can be verified directly from (45) and (49). The values of $\xi^{\prime \prime}$ at 0 and $\pm \frac{\pi}{2}$ can be computed via (46). By (47), $\left(\xi(t) \cos ^{2} t\right)^{\prime}=4 t \cos ^{2} t$. Therefore $\xi(t) \cos ^{2} t=\int_{\frac{\pi}{2}}^{t} 4 s \cos ^{2} s d s$, and

$$
\begin{aligned}
& \int_{-\frac{\pi}{2}}^{\frac{\pi}{2}} \xi(t) d t=2 \int_{0}^{\frac{\pi}{2}} \xi(t) d t=-8 \int_{0}^{\frac{\pi}{2}}\left(\frac{1}{\cos ^{2}(t)} \int_{t}^{\frac{\pi}{2}} s \cos ^{2} s d s\right) d t \\
= & -8 \int_{0}^{\frac{\pi}{2}}\left(\int_{0}^{s} \frac{1}{\cos ^{2}(t)} d t\right) s \cos ^{2} s d s=-8 \int_{0}^{\frac{\pi}{2}} s \cos s \sin s d s=-\pi .
\end{aligned}
$$

It is easy to see that $q$ and $q^{\prime}$ satisfy the following equations

$$
\frac{1}{2} q^{\prime \prime} \cos t-2 q^{\prime} \sin t-2 q \cos t=-4 \sin t,
$$


and

$$
\frac{\cos ^{2} t}{2\left(1+\cos ^{2} t\right)}\left(q^{\prime}\right)^{\prime \prime}-\frac{2 \cos t \sin t}{1+\cos ^{2} t}\left(q^{\prime}\right)^{\prime}-2\left(q^{\prime}\right)=-\frac{4}{1+\cos ^{2} t} .
$$

The last equation implies $q^{\prime}=\xi^{\prime \prime}$ cannot achieve its non-positive local minimum at a point in $\left(-\frac{\pi}{2}, \frac{\pi}{2}\right)$. On the other hand, $\xi^{\prime \prime}\left( \pm \frac{\pi}{2}\right)=2$, by equation (46), $\xi\left( \pm \frac{\pi}{2}\right)=0$ and $\xi^{\prime}\left( \pm \frac{\pi}{2}\right)= \pm \frac{2 \pi}{3}$. Therefore $\xi^{\prime \prime}(t)>0$ on $\left[-\frac{\pi}{2}, \frac{\pi}{2}\right]$ and $\xi^{\prime}$ is increasing. Since $\xi^{\prime}(t)=0$, we have $\xi^{\prime}(t)<0$ on $\left(-\frac{\pi}{2}, 0\right)$ and $\xi^{\prime}(t)>0$ on $\left(0, \frac{\pi}{2}\right)$. Similarly, from the equation

$$
\begin{gathered}
\frac{\cos ^{2} t}{2\left(1+\cos ^{2} t\right)}\left(q^{\prime \prime}\right)^{\prime \prime}-\frac{\cos t \sin t\left(3+2 \cos ^{2} t\right)}{\left(1+\cos ^{2} t\right)^{2}}\left(q^{\prime \prime}\right)^{\prime}-\frac{2\left(5 \cos ^{2} t+\cos ^{4} t\right)}{\left(1+\cos ^{2} t\right)^{2}}\left(q^{\prime \prime}\right) \\
=-\frac{8 \cos t \sin t}{\left(1+\cos ^{2} t\right)^{2}}
\end{gathered}
$$

we get the results in the last line of the lemma.

Set $h(t)=\xi^{\prime \prime}(t) t-\xi^{\prime}(t)$. Then $h(0)=0$ and $h^{\prime}(t)=\xi^{\prime \prime \prime}(t) t>0$ in $\left(0, \frac{\pi}{2}\right)$. Therefore $\left(\frac{\xi^{\prime}(t)}{t}\right)^{\prime}=\frac{h(t)}{t^{2}}>0$ in $\left(0, \frac{\pi}{2}\right)$. Note that $\frac{\xi^{\prime}(-t)}{-t}=\frac{\xi^{\prime}(t)}{t}$, $\left.\frac{\xi^{\prime}(t)}{t}\right|_{t=0}=\xi^{\prime \prime}(0)=2\left(3-\frac{\pi^{2}}{4}\right)$ and $\left.\frac{\xi^{\prime}(t)}{t}\right|_{t=\pi / 2}=\frac{4}{3}$. This completes the proof of the lemma.

\section{References}

[1] I. Chavel, Eigenvalues in Riemannian Geometry, Academic Press, Orlando, Fla, 1984.

[2] P. Li, Lecture Notes on Geometric Analysis, Lecture Notes Series, \#6, Seoul National University, Seoul, Korea.

[3] P. Li and A. Treibergs, Applications of Eigenvalue Techniques to Geometry, H. Wu, editor, Contemporary Geometry: J. Q. Zhong Memorial Volume Plenum, 21-52, 1991.

[4] P. Li and S. T. Yau, On the Schrödinger equation and the eigenvalue problem, Comm. Math. Phys, 88(1983), 309-318.

[5] P. Li and S. T. Yau, Estimates of eigenvalues of a compact Riemannian manifold, AMS Proc. Symp. Pure Math., 36(1980), 205-239.

[6] A. Lichnerowicz, Geometrie des groupes de tansformations, Dunod, Paris, 1958. 
[7] J. Ling, A lower bound for the gap between the first two eigenvalues of Schrödinger operators on convex domains in $\mathrm{S}^{n}$ or $\mathrm{R}^{n}$, Michigan Math. J., 40(1993), 259-270.

[8] J. Ling, A bound for the first fundamental gap. Ph.D. Dissertation, State University of New York at Buffalo.

[9] J. Ling, Estimates on the lower bound of the first gap, Preprint, 2004.

[10] R. Reilly, Applications of the Hessian operatior in a Riemannian manifold, Indiana Univ. Math. J., 26(1977), 459-472.

[11] R. Schoen, and S. T. Yau, Lecture Notes on Differential Geometry, Conference Procedings and Lecture Notes in Geometry and Topology, Vol 1, International Press, 1994.

[12] D. Yang, Lower bound estimates on the first eigenvalue for compact manifolds with positive Ricci curvature, Pacific Journal of Mathematics, 190(1999), 383-398.

[13] S. T. Yau, editor, Problem Section, Seminar on Diff. Geom., Princeton University Press, Princeton, 1982.

[14] J.-Q. Zhong and H. C. Yang, On the estimate of the first eigenvalue of a compact Riemannian manifod, Sci. Sinica, Ser. A 27, (1984), $1265-1273$.

Department of mathematics, Utah Valley State College, Orem, Utah 84058

E-mail address: lingju@uvsc.edu 